\title{
No Reference Objective Video Quality Measurement Based on Human Visual System
}

\author{
Subrahmanyam.Ch ${ }^{1}$, Dr.D.Venkata Rao ${ }^{2}$, Dr.N.Usha Rani ${ }^{3}$ \\ ${ }^{I}$ (Research Scholar, Dept of ECE, Vignan University, Guntur, India) \\ ${ }^{2}$ (Principal, Narasaraopeta Institute of Technology, Guntur, India) \\ ${ }^{3}$ (Head of the Department, Dept of ECE, Vignan University, Guntur, India)
}

\begin{abstract}
We tested how to improve the quality of the reconstructed video, several deblocking algorithms have been used. In this paper, we will introduce these deblocking algorithms and classify them into several categories. On the other hand, the conventional PSNR is widely adopted for estimating the quality of the compressed video. However, PSNR sometimes does not reveal the quality perceived by human visual system (HVS). In this paper, we will introduce one measurement to estimate the blockiness in the compressed video.
\end{abstract}

Keywords: - reconstructed video, deblocking algorithm, PSNR, HVS

\section{INTRODUCTION}

Block-based transform coding is popularly used in video compression standards such as MPEG and H.26x because of its excellent energy compaction capability and low hardware complexity. These standards achieve good compression ratio and quality of the reconstructed video when the quantizer is not vey coarse; however, in very low bit rate, the well-known annoying artifact in video compression coding come into existence and degrade the quality seriously. This artifact is called Blocking Artifact, which results from coarse quantization that discards most of the high frequency components of each segmented macro block of the original image and video frame and introduces severe quantization noise to the low frequency component. In order to reduce the annoying blocking artifacts, several deblocking algorithms had been proposed. We can classify the deblocking algorithms into four types: in-loop filtering, post-processing, pre-processing and overlapped block methods. The in-loop filtering algorithm inserts deblocking filter into the encoding and decoding loop of the video CODEC, and one example of this method is adopted in H.264/AVC. The postprocessing algorithms apply some post-processing low pass filters and algorithms after the image and video has been decoded to improve the image and video quality. The pre-processing algorithms pre-process the original image and video so that the quality of the reconstructed image and video can be the same as that without being processed under lower bit rate. The overlapped block methods include lapped orthogonal transform (LOT) whose transform bases are overlaid to each other and overlapped block motion compensation (OBMC) which consider the neighboring blocks for motion estimation and motion compensation in video coding.

\section{MASKING EFFECT OF HUMAN VISUAL SYSTEM}

There are three major observations on blocking artifacts could be noted in block-based transform coding (BTC).

I. Because of the masking effect of the human visual system (HVS), there are different sensitivity of the HVS to areas of the image and video with different complexity. The blocking artifacts are more noticeable in flat areas than in complex areas.

II. The deblocking filter can remove some high frequency discontinuity over the block boundaries; however, it may result into blurring the real edges in the original image or video frames.

III. The motion compensation prediction (MCP) propagates the blocking artifacts into the next frame in video coding.

We know that the blocking artifact will propagate into the later frames in video coding, which will degrade the visual quality and thus affect the compression ratio. Based on this observation, we can achieve higher compression ratio and better visual quality if we can effectively eliminate the blocking artifacts. Therefore, H.264/AVC and H.263+ add the deblocking filter into the coding loop to improve the visual quality and the accuracy of MCP.

According to the availability of a reference image, objective QA methods can be classified into three broad categories: full-reference QA methods, no-reference QA methods and reduced-reference QA methods. In full-reference QA methods, a test image is evaluated by comparing it with a reference image that is assumed to have perfect quality. The general used methods of full-reference quality assessment, which are called the error sensitivity based methods, consider the test image signal as the sum of the reference image 
signal and an error signal. One problem with these methods is that larger visible differences may not necessarily imply lower perceptual quality. Classical quality measures, such as MSE, WMSE or PSNR, are simple to calculate, have clear physical meanings, and are mathematically convenient in the context of optimization. But they are all error sensitivity based methods limitations, such as the supra-threshold problem, the natural image complexity problem. Hence, In this paper we prefer No-reference QA for Video.

\section{PROPOSED REFERNEC MODEL FOR VIDEO QUALITY MONITORING}

Three methodologies representing different measurement strategies for the assessment of the quality of video have been defined:

- methodology using the complete video reference (double-ended);

- methodology using reduced reference information (double-ended);

- methodology using no reference signal (single-ended).

The design and the development of a video quality monitor should consider a general structure of the measurement procedure for reduced reference and single-ended methodologies. The reference model is composed of the following four layers:

- Measurement methodology defines the class or the strategy relative to the application requirement;

- Measurement method is composed of a set of modules, algorithmic and associated ones, implemented to process inputs such as original signals or processed reference data, and provide output results such as processed reference data, level of impairment or final quality notation;

- Algorithmic module(s) is the basic block of signal processing functions composing the method. It composes the core of the method from which the final objective qualification is delivered;

- Associated module(s) is an additional function that aids the algorithmic module(s) in its operation by addressing such issues as dating, synchronization, presentation of data, etc.

Each one of those methodologies offers a specific approach relative to its operational use. Each methodology is well adapted to a specific field of applications. This is due to its technical implementation constraints:

- relevance of the measured data,

- availability of the reference,

- synchronization of original and impaired signals or data,

- transmission channel for reference data,

- real-time implementation,

- in-service use, etc.

Post-processing algorithms are the most popular methods for improving the quality of the image and video and eliminate the annoying blocking artifact. On the other hand, the post-processing algorithms can achieve deblocking without the original image and video, so the standard need not to be modified.

\section{Reduction of Blocking Artifacts in DCT Domain}

We introduce a post-processing filtering algorithm in DCT domain. We define the block $b_{m, n}^{k, l}(u, v)$ and $B_{m, n}^{k, l}(u, v)$ first. $b_{m, n}(u, v)$ is the (m,n)-th $8 \times 8$ block in the compressed image, and $B_{m, n}(u, v)$ is the DCT coefficients of $b_{m, n}(u, v) . b_{m, n}^{k, l}(u, v)$ is the shifted block with displacement $k$ pixel in the $x$ direction and displacement $l$ pixels in the $y$ direction with respective to block $b_{m, n}(u, v)$, and $B_{m, n}^{k, l}(u, v)$ is the DCT coefficients of the block $b_{m, n}^{k, l}(u, v)$. One example is shown in Fig. 1.

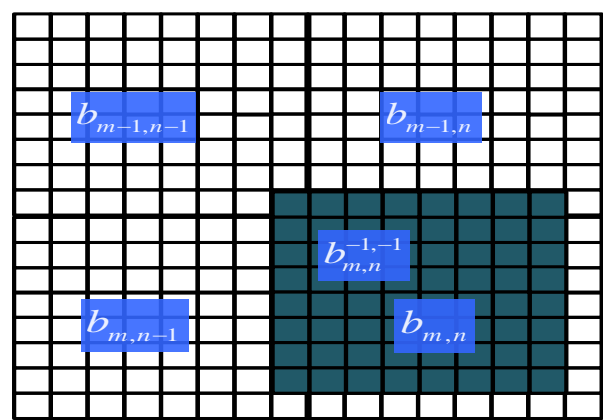

Fig. 1 Example of shifted block $b_{m, n}^{k, l}(u, v)$ 
In the original image, the neighboring DCT coefficients at the same frequency are very similar and do not vary radically within a small range. Thus, we can apply low pass filter to the DCT coefficients at each frequency to filter the high frequency parts resulting from blocking effect. However, this method may blur the real edges in the original image, so we must have the mechanism to detect activity of the block and apply the filter with corresponding strength.

DCT-domain filtering is applied to revise the block $B_{m, n}(u, v)$ to obtain the new DCT coefficients

$$
B_{m, n}(i, j) \text {. }
$$

$$
\begin{gathered}
B_{m, n}(i, j)=\frac{1}{W} \sum_{k=-h}^{h} \sum_{l=-h}^{h} w_{k, l} B_{m, n}^{k, l}(u, v) \\
W=\sum_{k=-h}^{h} \sum_{l=-h}^{h} w_{k, l}
\end{gathered}
$$

The post-filtering works in different ways for the blocks with different activities.

For blocks with low activity, the blocking artifact is more noticeable, so we apply strong filtering to smooth the high frequency components. The filter coefficients are defined.

$$
w_{k, l}=1, k, l=-2, \ldots, 2
$$

For blocks with high activity, the blocking artifact is less noticeable, so we apply filtering with less strength to smooth blocking artifact and preserve the real edge. The filter coefficients are defined.

$$
w_{k, l}=\left\{\begin{array}{l}
3, \text { for }(k, l)=(0,0) \\
1, \text { otherwise }
\end{array}\right.
$$

\section{EXPERIMENTAL RESULTS}

In this, we will show some experimental results. The image database used in the experiment is from LIVE, which consists of original high-resolution YUV format video files. It includes different distortion types of video sets. The detail of the video PSNR values are shown. We only use the total of 2 distorted video files in our experiment.

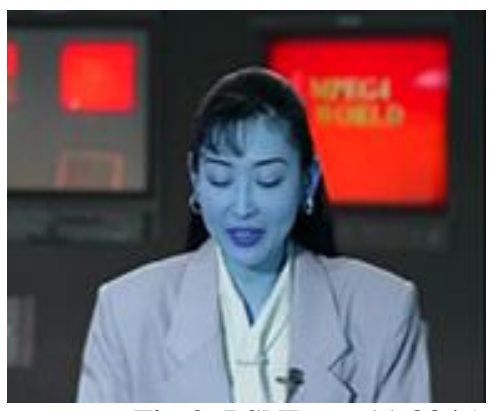

Fig.2. PSNR $=11.8345$

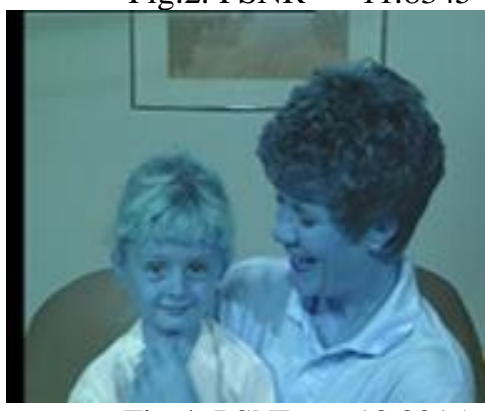

Fig.4. PSNR $=18.8015$

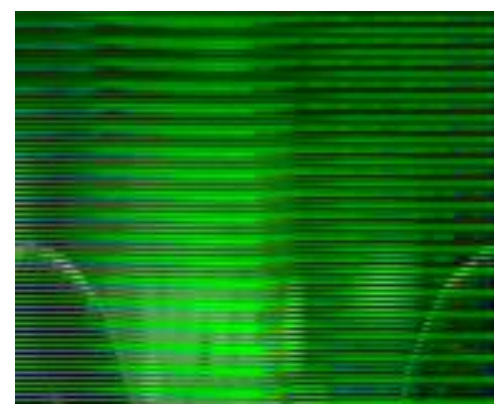

Fig.3. PSNR $=0.5926$

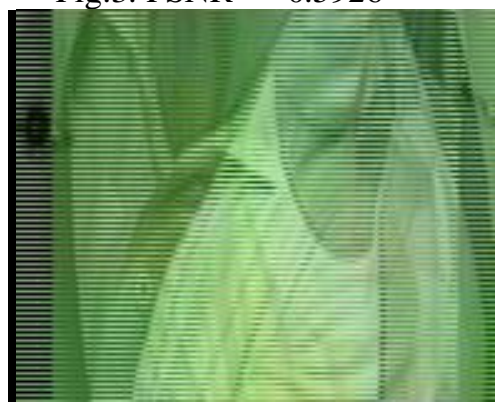

Fig.5. PSNR $=1.2104$

\section{CONCLUSION}

In our experiment, we use two different distortion types of video sets to evaluate the effectiveness and robustness of the proposed method. Compared with PSNR values with distorted video PSNR values, good results are obtained as shown in Fig. 2- Fig. 5. The major result of the proposed method is the possibility of 
evaluating video quality consistently with human perception for color images. Experimental evidence confirms that the proposed method provides a satisfactory approximation of the actual subjective scores.

\section{REFERENCES}

[1] B W Keelan. Handbook of Image Quality: Characterization and Prediction [M]. New York: Marcel Dekker Inc., 2002: 9.

[2] A. C Bovik. Introduction to Image and Video Quality Assessment[OL]. [2009-3-17]. http://live.ece.utexas.edu/research/quality/intro.htm.

[3] Z Wang, A. C Bovik, H R Sheikh, et al. Image Quality Assessment: From Error Visibility to Structural Similarity [J]. IEEE Trans. Image Process., 2004, 13(4): 600-612.

[4] C J van den Branden Lambrecht, Ed. Special Issue on Image and Video Quality Metrics [J]. Signal Process., 1998, 70(3): 155-294.

[5] VQEG. Final Report from the Video Quality Experts Group on the Validation of Objective Models of Video Quality Assessment [OL]. (2000-3-15) [2009-3-17]. http://www.its.bldrdoc.gov/vqeg/projects/frtv_phasel/COM-80E_final_report.pdf.

[6] T N Pappas and R J Safranek. Perceptual Criteria for Image Quality Evaluation. In: A. C Bovik, ed. Handbook of Image \&Video Processing [M]. San Diego: Academic Press, 2000: 669-684.

[7] Z Wang and A. C Bovik. Why is Image Quality Assessment so Difficult? [C]. Proc. of the IEEE ICASSP 2002, Orlando, Florida, 2002: IV-3313- IV-3316.

[8] Z Wang and A C Bovik. A Universal Image Quality Index [J]. IEEE Signal Processing Letters, 2002, 9(3): 81-84.

[9] G H Chen, C L Yang, L M Po, et al. Edge-based Structural Similarity for Image Quality Assessment ICASSP, Toulouse, France, 2006: II-933-II936.

[10] H R Sheikh, Z Wang, L Cormack, et al. LIVE Image Quality Assessment Database [OL]. (2005-213)[2009-3-17] http://live.ece.utexas.edu/research/quality/.

[11] Commission Internationale d'Eclairage. CIE Publication No.15.2, Colorimetry [S]. 2nd Ed. Vienna: CIE Central Bureau, 1986.

[12] K McLaren. An Introduction to Instrumental Shade Passing and Sorting and a Review of Recent Develop- ments [J]. J. Soc. Dyers Colour, 1976, 92(9): 317-326.

[13] Commission Internationale d'Eclairage. CIE Publication No. 116, Industrial Colour-Difference Evaluation [S]. Vienna: CIE Central Bureau, 1995.

[14] Commission Internationale d'Eclairage. CIE Publication No. TC1. 47, Hue and Lightness Correction to Industrial Colour Difference Evaluation [S]. Vienna: CIE Central Bureau, 2000.

[15] VQEG. Final Report from the Video Quality Experts Group on the Validation of Objective Models of Video Quality Assessment, Phase IVQEG [OL]. (2003-8-25) [2009-3-17]. http://www.its.bldrdoc.gov/vqeg/projects/frtv-_phaseII/downloads/VQEGII_Final_Report.pdf.

[16] H R Sheikh, M F Sabir, and A C Bovik. A Statistical Evaluation of Recent Full Reference Image Quality Assessment Algorithms [J]. IEEE Trans. Image Process., 2006, 15(11): 3441-3452. 